\title{
Do Auditory Temporal Discrimination Tasks Measure Temporal Resolution of the CNS?
}

\author{
Ian T. Zajac, Nicholas R. Burns \\ School of Psychology, University of Adelaide, Adelaide, South Australia. \\ Email:ian.zajac@adelaide.edu.au \\ Received July $1^{\text {st }}, 2011$; revised August $5^{\text {th }}, 2011$; accepted September $15^{\text {th }}, 2011$.
}

\begin{abstract}
Rammsayer \& Brandler (2002) have proposed that auditory temporal discrimination tasks provide a measure of temporal resolution of the CNS which is argued to be partly responsible for higher order cognitive functioning. We report on two studies designed to elicit the nature of the functions underpinning these auditory tasks. Study 1 assessed whether temporal generalisation (TG) might be better considered as a measure of working memory rather than of temporal resolution of the CNS. In $N=66$ undergraduates TG did not predict speed of processing tasks; however, there was evidence of a relationship between TG and working memory. Study 2 reanalyzed previously published data on temporal discrimination tasks and showed that the relationship between auditory temporal tasks and intelligence reflects memory functions and processing speed. Auditory temporal discrimination tasks are confounded by speed and memory and should not be considered as measures of temporal resolution of the CNS.
\end{abstract}

Keywords: Temporal Discrimination, Working Memory, Intelligence, Auditory Reaction Time

\section{Introduction}

The last few decades have seen a shift in focus from the taxonomic study of cognitive abilities to the identification of lower-order cognitive and physiological correlates of human intelligence (Neubauer \& Fink, 2005). This shift has been driven by a desire to identify the biological roots of higher order cognition (Stankov, 2005). The exploration of biological correlates of intelligence has been aided by advances in the measurement of brain activity. Studies employing electroencephalograms, for instance, have reported that peripheral nerve conduction velocity and event related potentials share variance with cognitive ability measures (Burns, Nettelbeck, \& Cooper, 2000; Reed \& Jensen, 1993). On the other hand, in order to measure lower-order cognitive processes, researchers have turned to a class of tasks termed Elementary Cognitive Tasks (ECTs). The impetus for this is that ECTs are characteristically easy tasks which putatively rely on a limited number of mental processes or operations (Carroll, 1993). Thus, they supposedly provide cleaner measures of biological processes than traditional, more complex tests (Stankov, 2005).

The two most commonly researched ECTs are reaction time (RT) and inspection time (IT): RT tasks measure the speed with which an individual is able to respond to a particular reaction stimulus; and IT tasks measure the minimum exposure duration required to accurately discriminate stimuli that differ on some dimension. Both classes of tasks are held to reflect information processing speed (Jensen, 2005). However, it has been found that performance in these tasks is relatively independent. The correlation between these ECTs is seldom more than $r=.30$, with the strength of the correlation appearing to increase as complexity of the RT task increases (Burns \& Nettelbeck, 2003; O'Connor \& Burns, 2003; Petrill, Luo, Thompson, \& Detterman, 2001).

Despite their relative independence, IT and RT tasks have been found to share a statistically significant amount of variance with measures of psychometric intelligence. People with higher speed of information processing - faster average RTs and shorter ITs - perform better on tests of cognitive ability than those who are slower. It has been proposed that RT and IT could account for as much as $25 \%$ of the variance in intelligence test performance (Grudnik \& Kranzler, 2001; Jensen, 1982, 2005, 2006; Nettelbeck, 1987, 2001, 2003). However, a more recent meta analysis which based its conclusions on 1146 correlations between speed of processing measures and intelligence measures proposes a much smaller effect: around 10\% shared variance for RT and intelligence and about $8 \%$ between IT and intelligence (Sheppard \& Vernon, 2008).

Regardless of the size of these effects, and returning to the idea of identifying the biological basis of intellectual functioning, it is necessary to explain the observed relationship between intelligence and performance in ECTs. Many explanatory models appeal to the concept of "neural efficiency" as the determinant of both information processing speed and intelligence (see e.g., Hendrickson, 1982; Hendrickson, 1982; Vernon, 1993). Jensen's (1982) model of neural oscillations, for example, proceeds from the assumption that RT provides an index of the efficiency of the central nervous system (CNS). Individual differences in both processing speed - as measured by ECTsand intellectual functioning are attributed to differences in the rate of oscillation between refractory and excitatory states of neurons. The transmission of neurally encoded information is assumed to be more efficient as well as faster at a higher rate of neural oscillations. This is because it takes less time for a neuron to re-enter its excitatory phase when processing information than when oscillations are slow.

An alternative theory linking higher-order cognitive processes to elementary functions has recently been revisited by Rammsayer and others (Helmbold \& Rammsayer, 2006; Helmbold, Troche, \& Rammsayer, 2006a, 2007a; Rammsayer \& Brandler, 2002, 2004, 2007). Originally proposed by Surwillo (1968), this theory also appeals to a hypothetical oscillatory, or "clock", mechanism in the CNS to explain individual differences in speed of information processing and intelligence. Thus, "if the hypothesised internal master clock of individual A 
works at half the clock rate as the one of individual B, then A does not only need twice as long as B to perform a specific sequence of mental operations, but also the occurrence probability of interfering incidents will be increased" (Rammsayer \& Brandler, 2007: p. 124); according to the theory this results in both slower performance on speed of processing tasks and lower intelligence. The central features of the internal clock mechanism are a pacemaker and an accumulator (Rammsayer \& Brandler, 2002).

Jensen (2006) cites findings from ECT research to support his neural oscillation model. In order to obtain empirical support for the master clock theory, Rammsayer and colleagues have sought to demonstrate that presumed measures of clock rate differ between individuals of low and high intelligence (Helmbold et al., 2006a, 2007a; Rammsayer \& Brandler, 2002, $2004,2007)$. They have argued that accuracy on psychophysical timing tasks - by analogy with performances on ECTsreflects basic processes related to neural efficiency (Helmbold, Troche, \& Rammsayer, 2007b). According to this theory the number of neural oscillations generated by the pacemaker during a timed interval is recorded by the accumulator and becomes the internal representation of that interval. Thus, the higher the frequency of oscillations the finer the temporal resolution.

Because audition has finer temporal resolution than vision (Rammsayer \& Brandler, 2002), attempts to measure temporal resolution of the CNS have focussed on auditory tasks. Rammsayer and Brandler (2002) found that auditory duration discrimination was significantly better for a high-IQ group than for a low-IQ group and that it explained around $20 \%$ of the total variance of a single fluid intelligence (Gf) measure. A later study concluded that a general pacemaker based interval timing mechanism is involved in auditory temporal order judgement, duration discrimination, and temporal generalisation and that performances on these tasks is independent of general auditory discrimination ability (Rammsayer \& Brandler, 2004). Factor scores on this general timing (Gt) mechanism have subsequently been shown to share substantial variance (about 25\%) with psychometric measures of general intellectual ability, otherwise referred to as " $g$ " (Rammsayer \& Brandler, 2007).

Whether Gt solely reflects temporal resolution is arguable and Helmbold et al. (2006a) have explored whether sensory discrimination abilities rather than temporal resolution of the CNS account for the relationship between Gt and intelligence. Temporal generalisation and pitch discrimination performance was measured and regression analyses showed these tasks combined to predict $25 \%$ of the variance in $g$ factor scores. The unique contributions of temporal and pitch tasks were $9 \%$ and $6 \%$, respectively. The shared, and presumably general, sensory processes accounted for the remaining $10 \%$ of predicted $g$. Helmbold et al. (2006a) concluded that the unique contribution of temporal discrimination to the prediction of $g$ supports the notion that it measures specific aspects of neuronal information processing related to intellectual capacity but independent of non-temporal aspects of sensory discrimination.

As noted, it has been proposed that auditory psychophysical timing tasks are analogous to existing ECTs in terms of measuring basic processes related to neural efficiency (Helmbold et al., 2007b). Therefore, one should expect these tasks to correlate at least moderately with existing ECTs, including RT and IT, but evidence regarding this hypothesis is equivocal (Helmbold et al., 2007a; Rammsayer \& Brandler, 2007). Although the correlation between latent RT and latent temporal discrimination factors appears moderately strong $(r=.65$ : Helmbold et al., 2007b), the correlation between individual temporal tasks and RT parameters is markedly weaker and in many cases not statistically significant. The average correlation between eight temporal tasks and different RT parameters in Helmbold and Rammsayer (2006) was only $r=-.19\left(S D_{r}=.07\right)^{1}$. This absence of significant correlations between temporal tasks and RT suggests that temporal discrimination tasks may not be measuring the elementary processes reflected in RT tasks.

Nonetheless, these findings have been interpreted as providing evidence that auditory temporal discrimination tasks index temporal resolution in the CNS. There are, however, several issues with these tasks which question whether the observed correlation between temporal performance and intelligence is a result of neural efficiency and, by extension, temporal resolution of the CNS.

First, although it has been supposed that these tasks are elementary (Rammsayer \& Brandler, 2004) some do not appear to be. The Temporal Generalisation (TG) task appears rather more complex than archetypal ECTs. TG requires participants to judge whether a test stimulus is the same as a standard stimulus learnt in a pre-exposure phase. Thus, the task requires: 1) accurate learning of the standard stimulus; 2) accurate registration of the test stimulus; 3 ) accurate retrieval of the learnt standard; and 4) a successful comparison of the test and learnt standard, in order to complete each test item. It appears that the cognitive operations required in this test are complex, and even if temporal resolution of the brain itself is independent of higher order cognitive operations (Rammsayer \& Brandler, 2002: p. 509), performance on this task is not likely to be. It is plausible that the observed relationship between TG and intelligence reflects the shared cognitive operations common to TG and general intelligence tests, rather than temporal resolution of the brain.

This hypothesis applies to other discrimination tasks used in these studies. Duration Discrimination (DD), for example, requires participants to compare two successively presented time intervals to decide which was longer. Thus, an internal representation of each interval must be formed and, given the length of the intervals - $1 \mathrm{sec}$ or longer in one condition - and the ISI $(900 \mathrm{~ms})$, these representations need to be accessible for up to three seconds after presentation; at least for the first presented interval. Unless the accumulator in the master clock theory incorporates an information storage component, performance on this task is also likely to rely on complex cognitive functions. Considering the requirements of both TG and DD, it appears that the cognitive operations involved may include substantial memory functions.

A second issue regarding these findings is that most have focused on factor scores. As such, little information is gleaned in terms of the relationship between specific temporal discrimination tests and intelligence measures. Moreover, the nature of the latent construct defined by the temporal tasks is merely surmised based on theory of what the tests have in common. In order to accurately assess whether internal clock rate - or temporal resolution of the CNS - is related to intellectual capacity, it must first be established that each of the individual tests used provides some measure of this. Only then can a latent variable defined by these tests be taken to represent the internal clock rate.

This paper presents the findings of two studies on temporal discrimination tasks. The impetus for these studies was to investigate whether temporal discrimination tasks provide a measure of elementary functions such as temporal resolution of

${ }^{1}$ These correlations were not reported in the published article and we are grateful to Prof. Rammsayer for providing these to us. 
the CNS, or whether they might better be conceptualized as measures of more complex cognitive operations like memory functions.

\section{Study 1}

The Temporal Generalisation (TG) task has been shown to relate to $g$ but its relationship with specific cognitive abilities and ECTs has not been considered. Thus, the relationship may reflect executive cognitive functions utilised in task performance and not neural efficiency, as proposed. The purpose of this study was to provide a test of this hypothesis by exploring the relationship between TG and measures of processing speed (Gs) and working memory (WM). Importantly, speed of processing was measured by traditional speed tasks and ECTs, including RT and IT, because of the considerable evidence that these ECTs are reliable measures of elementary functions (Jensen, 2006; Nettelbeck, 2001). If TG measures elementary functions as opposed to executive cognitive functions, then the relationship between TG and Gs will be stronger than that between TG and WM.

We used the same dissociation paradigm as Helmbold et al. (2006a). The purpose was to assess the direct relationship of TG to Gs after partialling out variance due to general sensory discrimination processes; reflected in the pitch discrimination task (APd). If TG measures elementary processes related to intelligence and which are independent of general sensory discrimination, then TG should make a direct contribution to the prediction of intelligence test performance.

The superior temporal discrimination in audition has been the motivation for the use of auditory tasks. However, if the master clock which determines performance on these tasks is a general feature of the neural system, it should also be responsible for temporal discrimination in other modalities. Therefore, the current study sought also to measure temporal resolution of vision. This was achieved through adapting the dissociation paradigm for the visual system to include a temporal and a line-length discrimination task. Line-length discrimination ability would be measured to assess variance in visual temporal performance reflecting general sensory processing. The correlation between the visual and auditory temporal discrimination tasks should be at least moderately strong if they reflect the same elementary timing processes.

Rammsayer and Brandler (2002) have reported that temporal resolution of the CNS is independent of cognitive operations. We tested this assumption by introducing a backward masking condition for the discrimination tasks. Masking has previously been used in visual and auditory modalities to investigate temporal processes underpinning perception, and which operate at a precognitive level (Breitmeyer, 2007). If temporal tasks measure temporal resolution of the CNS then their relationship with cognitive ability measures should not be negatively affected by the introduction of a masking stimulus; because it emphasises pre-cognitive functioning. In fact, the strength of the relationships might be expected to increase.

\section{Methods}

\section{Participants}

The Participants were $\mathrm{N}=66$ undergraduate students of the University of Adelaide, South Australia. There were 7 males and 26 females in each of the masked and unmasked conditions. All participated as part of their Level I Psychology course requirements.

\section{Apparatus}

The presentation of all tasks and recording of responses was controlled by one of two identical computers. Visual stimuli were presented on 17 inch LCDs. Auditory stimuli were presented via Sony MDR-XD100 stereo headphones. Auditory tones were calibrated prior to the study using a Radio Shack 33 4050 Sound Level Meter.

\section{Discrimination Tasks}

Auditory and visual discrimination abilities were assessed using the experimental dissociation paradigm developed by Gibbons, Brandler, \& Rammsayer (2002); stimuli varied on two dimensions simultaneously. The first dimension was temporal: there were seven levels of stimulus duration. The second dimension for the auditory modality was pitch and for the visual modality was line-length; there were seven levels of each (see Appendix A). Line length dimensions were piloted on a small number of colleagues to be at a comparable level of difficulty to the duration levels.

The design of the set of stimuli for the dissociation paradigm is based on the requirement that: 1) for duration, as well as pitch/line length, there should be a probability for the standard stimulus of .33 in the total number of trials; 2) within each level of one stimulus dimension, each level of the other dimension should be represented; and 3) for each of the seven levels of one stimulus dimension, there should be a probability of .33 for the occurrence of the standard of the other stimulus dimension. Simultaneous variation on two dimensions according to these requirements results in a set of 81 stimuli for each of the visual and auditory tasks, resulting in the frequency distribution presented in Appendix A. The test phase for each of the discrimination tasks comprised 81 trials, including 27 presentations of the standard and nine presentations of each nonstandard stimulus. Presentation order within each task was pseudo-randomised, with the restriction that there were no more than two successive presentations of the standard. The outcome measure for each of the discrimination tasks was percentage of standard stimuli correctly identified.

Auditory Temporal and Pitch Discrimination Tasks. In each task, participants were required to identify the standard tone among the set of nonstandard tones. Participants were instructed to attend solely to tone duration in the temporal task, and to tone frequency in the pitch task. All tones were presented at an intensity of $67 \mathrm{db}$. Each task was preceded by a learning phase in which participants were asked to learn the standard tone. For the temporal task, a standard tone duration (i.e., $200 \mathrm{~ms})$ with a pitch $(900 \mathrm{~Hz})$ not administered during the test phase was presented five times. For the pitch task, the learning phase consisted of five presentations of the standard tone (i.e., $1000 \mathrm{~Hz}$ ) for $260 \mathrm{~ms}$, a duration which was not included in the test period. The testing phase immediately followed and the onset of each trial was marked by the presentation of a visual fixation point (small white cross) in the centre of the computer screen. After a foreperiod of $1000 \mathrm{~ms}$ the trial stimulus was presented and the cross remained on the screen. In the masking condition, a burst of white noise immediately followed the trial stimulus for $500 \mathrm{~ms}$, otherwise the trial terminated. Following each trial the participant mouse-clicked one of the onscreen buttons ("standard" or "nonstandard") to indicate whether they thought the trial stimulus matched the frequency or duration of the standard tone, depending upon which task was being completed. Feedback was given for each trial in the form of a "correct" or "incorrect" on-screen message which was displayed for $500 \mathrm{~ms}$. Subsequent trials commenced im- 
mediately after the feedback.

Visual Temporal and Line-Length Discrimination Tasks. The requirements of these tasks were similar to the auditory tasks. White horizontal lines presented against a black computer screen were used analogously to tones in the auditory tasks. For the temporal task, participants were asked to attend solely to stimulus duration whilst in the line-length task they were asked to attend solely to the length of the line. The learning phase for the temporal task consisted of five presentations of the standard duration (i.e., $200 \mathrm{~ms})$ with a line length $(6 \mathrm{~cm})$ not administered during the test period and for the line-length task, consisted of five presentations of a standard $10 \mathrm{~cm}$ line for a duration $(260 \mathrm{~ms})$ not included in the test. The testing phase immediately followed and each trial was marked by the onset of a visual fixation point (small white cross) in the centre of the computer screen. After a foreperiod of $1000 \mathrm{~ms}$ the visual fixation point was replaced by the trial stimulus. In the masking condition, a $4 \times 8$ grid of $16 \mathrm{~cm}$ wide by $6 \mathrm{~cm}$ high lines immediately followed the trial stimulus for $500 \mathrm{~ms}$ (see Figure 1) otherwise the trial terminated. The response format was the same as for the auditory tasks with participants indicating whether they thought the test stimulus matched the duration or line-length of the standard.

\section{Working Memory Task}

Dot Matrix Test (DM). A computer-administered version of the Dot Matrix Test (Law et al., 1995) was used as a measure of working memory (WM). Participants verified a series of simple matrix equations whilst simultaneously remembering the locations of dots on a $5 \times 5$ grid. Matrix equations were either addition or subtraction equations presented as lines drawn on $3 \times 3$ dot matrices. Participants verified each equation by mouseclicking either the "True" or "False" buttons displayed on the screen within 10 seconds, otherwise they received a prompt ("response required"). Following an incorrect response the message "No, look again closely" was displayed, and the equation remained until a correct was response was given.

Following correct responses a $5 \times 5$ grid was displayed for $1500 \mathrm{~ms}$ with a dot presented in one of the squares. There were four levels during the test $(2,3,4$, and 5 equation-grid pairs each with 4 items) and this equation-grid sequence was repeated according to the level. At the end of each equation-grid sequence, a blank $5 \times 5$ grid was displayed on the screen. Participants were required to mouse-click the spaces on the blank grid which had contained the dots during the trial sequence. Participants could not select more grid spaces than there were equation-grid pairs but they could select fewer grid spaces (e.g., 3 of 5 dot locations). An "enter" button was clicked after locations were selected. Three practice questions consisting of two equation-grid pairs preceded the test. The measure for the task was the number of dot positions, out of a total of 56, correctly recalled.

\section{Speed of Processing}

Symbol Digit (SD). A computerised coding task was em-
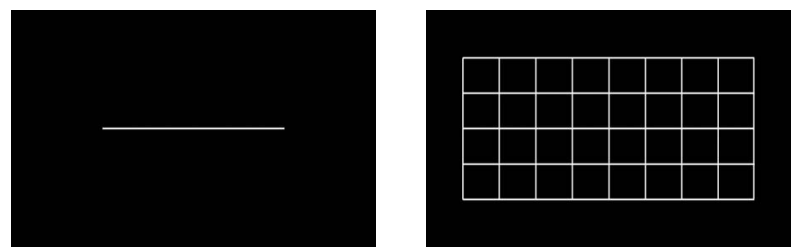

Figure 1.

Target and masking stimuli used in the visual discrimination tasks. ployed as a measure of Gs (see McPherson \& Burns, 2005, for a detailed description of this task). A code table was presented at the top of the computer screen throughout the task. This comprised of nine symbols arranged horizontally, to which nine digits were paired; digits were presented directly beneath the symbols so that they were aligned. For each item, one symbol was presented in the centre of the computer screen and participants responded by left clicking the mouse on its corresponding digit in a $3 \times 3$ numerical grid positioned at the bottom of the screen. Subsequent items did not commence until a correct response was registered. Participants were required to complete two practice trials correctly before they proceeded to the test. The outcome measure was the number of items correctly completed in 120 seconds

Audio Code $(A C)$. This task was developed in our laboratory to be an auditory analogue of the symbol digit task described above. It has good reliability $(r=.89)$ and correlates well with other speed measures. In this task, a code table is displayed at the top of the computer screen for the duration of the task. This comprised of pictures of eight musical instruments arranged horizontally, to which one of the numbers one through eight was paired; the instruments were a snare, trumpet, guitar, cymbals, piano, bell, harp and violin. For each item, the sound of one of the instruments was presented via headphones at an intensity of $65 \mathrm{db}$. Participants responded by left clicking the mouse on its corresponding digit in a $2 \times 4$ numerical response grid positioned at the bottom of the screen. Subsequent items commenced after a response was registered. Participants were required to complete four practice trials correctly before they proceeded to the test. The outcome measure was the number of items correctly completed in 120 seconds.

Visual Inspection Time (VIT). Stimuli were presented on a video monitor at a viewing distance of approximately $60 \mathrm{~cm}$ Preceding the target figure was a warning cue of approximately $520 \mathrm{~ms}$; the cue was a small white plus $(+)$ sign measuring $6 \times$ $6 \mathrm{~mm}$, presented in the centre of the computer screen. The target figure consisted of two vertical lines; one measured $15 \mathrm{~mm}$ and the other $30 \mathrm{~mm}$. These were joined at the top by a horizontal line of approximately $18 \mathrm{~mm}$. A "flash mask" (see Evans \& Nettelbeck, 1993) of $375 \mathrm{~ms}$ immediately replaced the target figure and consisted of two vertical lines $35 \mathrm{~mm}$ in length, shaped as lightning bolts. The shorter line appeared on either side of the target figure equiprobably.

A computerised tutorial preceded the test phase and the instructions emphasised accuracy rather than speed of responding. What was required was explained using diagrams, along with unmasked target stimuli. Practice trials required 10 correct trials out of 10 with a stimulus onset asynchrony (SOA) of approximately $835 \mathrm{~ms} ; 10$ correct trials out of 10 with SOA approximately $420 \mathrm{~ms}$; and nine correct trials out of 10 with SOA approximately $250 \mathrm{~ms}$. The estimation process began with SOA approximately $250 \mathrm{~ms}$ and followed an adaptive staircase algorithm (Wetherill \& Levitt, 1965). The algorithm required three correct responses at any SOA before SOA was reduced by approximately $17 \mathrm{~ms}$. The average SOA was calculated over eight reversals of direction on the staircase, giving an estimate of the SOA with an associated probability of $79 \%$ of making a correct response. Participants indicated on which side the short line appeared by clicking either the left or right mouse button, respectively.

Auditory Reaction Time (ART). This task required participants to respond as quickly as possible to an auditory target stimulus. To start each trial, the participant pressed the number "5" key in the numeric keypad on the computer keyboard. After 
$300 \mathrm{~ms}$ a short beep $(100 \mathrm{~ms}$ at $880 \mathrm{~Hz})$ was presented to confirm the trial had started. The target stimulus was then presented after a silent interval of variable duration $(1300 \mathrm{~ms}$, $1700 \mathrm{~ms}, 2100 \mathrm{~ms}, 2500 \mathrm{~ms}$ ), and it was a $500 \mathrm{~ms}$ "bell" sound centered on a frequency of $800 \mathrm{~Hz}$. Participants were instructed to lift their finger off the number " 5 " key when they heard the target sound and press the number " 8 " key in the numeric keypad as fast and as accurately as possible. The test phase consisted of 32 trials before which participants had to complete five practice trials correctly. Mean RT was calculated after removing errors and outliers $( \pm 3 \mathrm{SD})$. The average number of trials remaining after these removals - and from which Mean $\mathrm{RT}$ was derived-was $\mathrm{M}=31.30(\mathrm{SD}=.63, \mathrm{Min}=30, \mathrm{Max}=$ 32).

\section{Procedure}

Upon attending the testing session participants were assigned to either the masked or unmasked condition depending on whether they were an odd or even numbered participant. They were seated in a quiet room in the laboratory and were guided through the tasks by the computer. The four discrimination tasks (see below) were interspersed with cognitive ability measures, which were ordered as they are set out below. The discrimination tasks were ordered so as to switch between modalities (auditory pitch/visual length/auditory temporal/visual temporal), and the discrimination tasks were counterbalanced within conditions to reduce fatigue effects (visual length/auditory pitch/visual temporal/auditory temporal). The ordering of cognitive ability measures remained constant. The testing session took 60 minutes to complete.

\section{Results}

After collating the data it was apparent that two participants did not complete Dot Matrix (DM), one participant failed to complete Auditory Temporal discrimination $\left(\mathrm{AT}_{d}\right)$ and another participant failed to complete Symbol Digit (SD). These missing data were replaced using the Expectation Maximization (EM) method in Missing Values Analysis in SPSS v.15. Following this an outlier analysis was performed by standardizing scores on each variable. The only identified outlier was for Audio Code ( $\mathrm{AC} ; \mathrm{z}=3.14)$, which was deleted and subsequently replaced using EM.

Descriptive statistics for the cognitive measures and discrimination tasks are presented in Table 1 . As can be seen, performance in the masked condition was poorer for all of the discrimination tasks, with small to large effects. The difference was only statistically significant for the auditory temporal discrimination task $\left(\mathrm{AT}_{d}[\mathrm{t}(64)=2.11, p=.038]\right)$ and auditory pitch discrimination task $\left(\mathrm{AP}_{d}[\mathrm{t}(64)=2.77, p=.007]\right)$.

Table 2 presents the correlations between the cognitive tests for the total sample and Table 3 displays the correlations between the discrimination tasks for the masked and un-masked conditions. As can be seen the correlations between the cognitive tests are small-to-moderate and the correlations between the discrimination tasks are moderate-to-strong. Of particular note is the correlation between $\mathrm{AT}_{d}$ and Visual Temporal $\left(\mathrm{VT}_{d}\right)$ discrimination. As hypothesized, the correlation between them is notably strong indicating that to a large degree these tasks index the same construct.

In order to assess the extent to which the temporal tasks predict performance in the speed tasks and working memory task (DM), linear regression was used. Rather than regress each of the speed tasks onto the discrimination tasks, independently, a composite speed measure was calculated by averaging stan-
Table 1.

Descriptive statistics for discrimination tasks, cognitive measures, VIT and $R T$.

\begin{tabular}{llccccc}
\hline & & $\mathrm{M}^{\mathrm{a}}$ & $\mathrm{SD}$ & Min & Max & $\mathrm{d}^{\mathrm{b}}$ \\
\hline $\mathrm{VT}_{d}$ & Unmasked & .58 & .18 & .07 & .89 & \\
& Masked & .52 & .19 & .15 & .89 & .34 \\
\multirow{2}{*}{$\mathrm{AT}_{d}$} & Unmasked & .68 & .16 & .26 & .93 & .53 \\
& Masked & .58 & .19 & .11 & .89 & \\
$\mathrm{VL}_{d}$ & Unmasked & .81 & .13 & .52 & 1 & .33 \\
& Masked & .77 & .11 & .56 & .96 & \\
$\mathrm{AP}_{d}$ & Unmasked & .64 & .15 & .26 & .89 & .71 \\
& Masked & .53 & .16 & .19 & .81 & \\
$\mathrm{SD}$ & & 90.8 & 16.1 & 64 & 133 & \\
$\mathrm{AC}$ & & 63.8 & 7.9 & 49 & 82 & \\
$\mathrm{DM}$ & & 38 & 6.3 & 19 & 51 & \\
$\mathrm{VIT}(\mathrm{ms})$ & & 45.3 & 11.6 & 19.5 & 76.4 & \\
$\mathrm{ART}(\mathrm{ms})$ & & 502.6 & 115.4 & 312.4 & 768.1 & \\
\hline
\end{tabular}

$\mathrm{VT}_{d}=$ Visual Temporal Discrimination; $\mathrm{VL}_{d}=$ Visual Length Discrimination; $\mathrm{AP}_{d}=$ Auditory Pitch Discrimination; $\mathrm{AT}_{d}=$ Auditory Temporal Discrimination; $\mathrm{SD}=$ Symbol Digit AC = Audio Code DM = Dot matrix $;$ VIT $=$ Visual Inspection Time; ART $=$ Auditory Reaction Time. ${ }^{\text {a }}$ Temporal tasks $=$ percent correct; $\mathrm{SD}, \mathrm{AC} \& \mathrm{DM}=\mathrm{N}$ correct; $\mathrm{VIT}$ and $\mathrm{RT}=$ msec, ${ }^{\mathrm{b}}$ Cohen's $\mathrm{d}$.

Table 2.

Correlations between discrimination tasks for masked (above diagonal) and unmasked conditions (below diagonal).

\begin{tabular}{lcccc}
\hline & $\mathrm{VT}_{d}$ & $\mathrm{VL}_{d}$ & $\mathrm{AT}_{d}$ & $\mathrm{AP}_{d}$ \\
\hline $\mathrm{VT}_{d}$ & - & $.36^{*}$ & $.65^{* *}$ & .27 \\
$\mathrm{VL}_{d}$ & $.34^{*}$ & - & $.35^{*}$ & .17 \\
$\mathrm{AT}_{d}$ & $.64^{* *}$ & .20 & - & $.50^{* *}$ \\
$\mathrm{AP}_{d}$ & $.59^{* *}$ & $.30^{*}$ & $.66^{* *}$ & - \\
\hline
\end{tabular}

$\mathrm{VT}_{d}=$ Visual Temporal Discrimination; $\mathrm{VL}_{d}=$ Visual Length Discrimination; $\mathrm{AP}_{d}=$ Auditory Pitch Discrimination; $\mathrm{AT}_{d}=$ Auditory Temporal Discrimination ${ }^{*} p<.05$ (1-tailed) ${ }^{* *} p<.01$ (1-tailed)

Table 3.

Correlations between cognitive tests.

\begin{tabular}{lcccc}
\hline & SD & AC & DM & VIT \\
\hline AC & $.47^{* *}$ & & & \\
DM & $.25^{*}$ & $.34^{* *}$ & & \\
VIT & -.17 & -.15 & $-.24^{*}$ & \\
ART & $-.39 * *$ & $-.21^{*}$ & -.20 & .00 \\
\hline
\end{tabular}

$\mathrm{SD}=$ Symbol Digit AC = Audio Code DM = Dot matrix $;$ VIT $=$ Visual Inspection Time; ART = Auditory Reaction Time. ${ }^{*} p<.05$ (1-tailed) ${ }^{* *} p<.01$ (1-tailed).

dardized scores on these variables (SD, AC, VIT, ART). A series of models were subsequently run in which either the composite speed measure or working memory measure (DM) was the dependent variable. The visual discrimination or auditory discrimination tasks were used as independent/predictor 
variables.

The results of these analyses are presented in Table 4. As can be seen, none of the models was statistically significant. The association between discrimination tasks and DM does however appear to be stronger than for the composite speed measure as well as more consistent. It is of a comparable magnitude in each of the modalities and in the different masking conditions. Because the discrimination tasks are effectively identical in both conditions - they differed only in terms of the addition of a backward-masking stimulus - the regressions with DM as the dependent variable were repeated using the total sample. According to these models, visual discrimination tasks and auditory discrimination tasks predicted a statistically significant amount of variance in DM (visual model $\left[R^{2}=.09, F(2,63)=\right.$ $3.26, p=.045]$ and auditory model $\left[R^{2}=.11, F(2,63)=3.74, p\right.$ $=.029])$, and the sizes of the effects remained consistent with those in Table 5. The standardized coefficients for the auditory temporal and pitch tasks were $\beta=.30$ and $\beta=.04$, and $\beta=.22$ and $\beta=.15$ for the visual temporal and line length tasks. Thus, in both modalities the temporal task is the stronger predictor of DM.

\section{Discussion-Study 1}

The relationship between Temporal Generalisation (TG) and markers of specific cognitive abilities was explored. The analyses suggest TG relates more strongly to the marker of Working Memory (WM) than to the composite speed measure. This result provides only limited support for the hypothesis that TG measures executive cognitive functions and not temporal resolution of the CNS because of the lack of statistical power and the limited number of marker tests.

\section{Study 2}

In light of the limited evidence provided in Study 1, the purpose of the current study was to explore further whether temporal tasks rely on memory functions by reanalysing previously published data. Rammsayer and Brandler (2007) reported on five temporal discrimination tasks; the Hick RT task (Hick, 1952); and a well defined battery of cognitive ability tasks measuring different aspects of intelligence corresponding to Thurstone's (1938) primary mental abilities. These tasks were

Table 4.

Regression models for masked and unmasked conditions.

\begin{tabular}{ccccccc}
\hline DV & IV & Condition & $\mathrm{R}^{2}$ & $\mathrm{~F}$ & $\mathrm{df}$ & $\mathrm{p}$ \\
\hline & $\mathrm{VT}_{\mathrm{d}} \& \mathrm{VL}_{\mathrm{d}}$ & Masked & .02 & .22 & 2,30 & 0.80 \\
$\begin{array}{c}\text { Composite } \\
\text { Speed }\end{array}$ & & Unmaksed & .02 & .23 & 2,30 & 0.80 \\
& $\mathrm{AT}_{\mathrm{d}} \& \mathrm{AP}_{\mathrm{d}}$ & Masked & .02 & .33 & 2,30 & 0.72 \\
& & Unmasked & .09 & 1.51 & 2,30 & 0.24 \\
& $\mathrm{VT}_{\mathrm{d}} \& \mathrm{VL}_{\mathrm{d}}$ & Masked & .12 & 1.96 & 2,30 & 0.16 \\
& & Unmaksed & .08 & 1.33 & 2,30 & 0.28 \\
& & Masked & .09 & 1.45 & 2,30 & 0.25 \\
& $\mathrm{AT}_{\mathrm{d}} \& \mathrm{AP}_{\mathrm{d}}$ & Unmasked & .10 & 1.73 & 2,30 & 0.20 \\
\hline
\end{tabular}

$\mathrm{VT}_{d}=$ Visual Temporal Discrimination; $\mathrm{VL}_{d}=$ Visual Length Discrimination; $\mathrm{AP}_{d}=$ Auditory Pitch Discrimination; $\mathrm{AT}_{d}=$ Auditory Temporal Discrimination $\mathrm{DV}=$ Dependent Variable; IV = Independent Variable. completed by a large sample $(N=100)$. The temporal tasks included: 1) Duration Discrimination (DD), requiring a decision concerning which of two successively presented timed intervals was longer; 2) Rhythm Perception (RP), requiring a decision concerning which of five beat-to-beat silent intervals - marked by $3 \mathrm{~ms}$ clicks - deviated from the constant $150 \mathrm{~ms}$ duration; 3) Temporal-order Judgment (TOJ), in which participants decide whether the onset of a Visual LED preceded that of an auditory stimulus, or vice versa; 4) Auditory Flutter Fusion (AFF), which derives an estimates of the ISI at which two successively presented auditory noise bursts appear fused; and 5) Temporal Generalisation (TG). These tasks comprise the battery used in previous investigations of temporal discrimination (see Helmbold \& Rammsayer, 2006; Helmbold et al., 2007b; Rammsayer \& Brandler, 2002, 2004, 2007).

Rammsayer and Brandler (2007) reported that a temporal $g$ (Gt) factor defined by the discrimination tasks predicted $31 \%$ of variance in psychometric $g$, as defined by the cognitive ability measures. Combining Gt and a Hick $g$ factor increased the proportion of explained psychometric $g$ by only $2 \%$. The unique contribution of temporal $g$ was $20.5 \%$, the shared contribution of temporal and Hick $g$ was $10.5 \%$, and the unique contribution of Hick $g$ was only $1.5 \%$. The authors concluded that temporal discrimination reflects an aspect of brain functioning that is stronger and more comprehensively related to $g$ than parameters derived from the Hick RT task.

As already noted, temporal discrimination tasks may invoke demands on executive cognitive functions. To the extent that this is so, one would expect a Gt factor to relate strongly with $g$ - and to a greater degree than RT tasks - because it would be saturated with variance reflecting cognitive functions underpinning both Gt and $g$. We have argued that the processes underpinning performance on temporal discrimination tasks might best align with memory functions, and previous research has established a strong and consistent relationship between WM and reasoning ability; as measured by intelligence tests (e.g. Burns, Nettelbeck, \& McPherson, 2009; Kyllonen \& Christal, 1990). Thus, tasks relying on memory functions should relate strongly to measures of intelligence, and temporal discrimination tasks may be an example of such tasks. Put more concisely, memory functions rather than temporal resolution of the CNS may be responsible for the relationship between temporal discrimination and intelligence. We present a reanalysis of Rammsayer and Brandler's (2007) data with the aim being to test whether memory mediates the relationship between temporal discrimination and intelligence.

\section{Methods}

Listed in Table 5 are the cognitive ability measures and temporal discrimination tasks used by Rammsayer and Brandler (2007) which are relevant to our aims. Participants in their study were 40 male and 60 female volunteers ranging in age from 18 to 45 years ( $M$ and $S D$ of age: $26.0 \pm 6.8$ years). The cognitive measures are composed of subtests of the Leistungsprüfsystem (Horn, 1983), Berliner Intelligenztruktur-Test (Jäger, Sü $\beta$, \& Beauducel, 1997), and the German adaptation of Cattell's Culture Free Test Scale 3 (CCFT; Cattell, 1961; Weiss, 1971). Three of these subtests measure memory functions (Verbal, Numerical and Spatial Memory). The temporal tasks and their requirements are described briefly above and more detailed explanations can be found in the original publication.

A data file containing the correlations, means and standard deviations reported in Rammsayer and Brandler (2007) was created for analysis using MPlus 5.2 (Muthen \& Muthen, 1998). 
Table 5 .

Intelligence scales and discrimination tasks used in Rammsayer and Brandler (2007) and the broad ability constructs measured.

\begin{tabular}{|c|c|c|c|}
\hline Intelligence Tests & $\begin{array}{l}\text { Broad } \\
\text { Ability }\end{array}$ & $\begin{array}{c}\text { Temporal } \\
\text { Discrimination Tests }\end{array}$ & $\begin{array}{l}\text { Broad } \\
\text { Ability }\end{array}$ \\
\hline $\begin{array}{c}\text { Verbal } \\
\text { Comprehension (VC) }\end{array}$ & Gc & $\begin{array}{c}\text { Duration } \\
\text { Discrimination (DD1) }\end{array}$ & Gt \\
\hline Word Fluency (WF) & Gc & $\begin{array}{c}\text { Duration } \\
\text { Discrimination (DD2) }\end{array}$ & Gt \\
\hline Perceptual Speed (PS) & Gs & $\begin{array}{c}\text { Duration } \\
\text { Discrimination (DD3) }\end{array}$ & Gt \\
\hline Number 1 (N1) & Gs & $\begin{array}{c}\text { Temporal } \\
\text { Generalisation (TG1) }\end{array}$ & Gt \\
\hline Number 2 (N2) & Gs & $\begin{array}{c}\text { Temporal } \\
\text { Generalisation (TG2) }\end{array}$ & Gt \\
\hline Space 1 (SP1) & Gf & $\begin{array}{c}\text { Rhythm } \\
\text { Perception (RP) }\end{array}$ & Gt \\
\hline Space 2 (SP2) & Gf & $\begin{array}{c}\text { Tonal-order } \\
\text { Judgment (TOJ) }\end{array}$ & Gt \\
\hline $\begin{array}{l}\text { Flexibility of } \\
\text { Closure (CLO) }\end{array}$ & Gf & $\begin{array}{l}\text { Auditory Flutter } \\
\text { Fusion }(\mathrm{AFF})^{\mathrm{a}}\end{array}$ & Gt \\
\hline Series (SE) & Gf & & \\
\hline Classifications (CL) & Gf & & \\
\hline Matrices (MA) & Gf & & \\
\hline Topologies (TO) & Gf & & \\
\hline Verbal Memory (VM) & $\mathrm{Gm}$ & & \\
\hline $\begin{array}{c}\text { Numerical } \\
\text { Memory (NM) }\end{array}$ & $\mathrm{Gm}$ & & \\
\hline Spatial Memory (SM) & $\mathrm{Gm}$ & & \\
\hline
\end{tabular}

Note: Gc, Crystallised Intelligence; Gs, General Speed of Processing; Gf, Fluid Intelligence; Gm, General Memory; Gt, General Temporal Discrimination, ${ }^{\mathrm{a}} \mathrm{AFF}$ excluded from study two analyses.

Confirmatory Factor Analysis (CFA) was then undertaken on the covariance matrix using Maximum Likelihood estimation. By using this approach, different models were able to be tested which either included or omitted a relationship between temporal discrimination and memory functions, and these were compared using the model chi-square difference test. The fit of CFA models was assessed using the chi-squared test of model fit $\left(\chi^{2}\right)$, the comparative fit index (CFI), the root mean squared error of approximation (RMSEA), and the standardized root mean squared residual (SRMR).

\section{Results}

We attempted to confirm the presence of the general timing (Gt) factor reported in Rammsayer and Brandler (2007). However, we excluded the Auditory Flutter Fusion (AFF) task from our analysis because it has typically loaded poorly on Gt and might better be considered a sensory rather than temporal measure. CFA results indicate that the temporal tasks defined a Gt factor adequately $\left[\chi^{2}(14)=20.26, p=.122\right.$; CFI $=.965$; RMSEA $=.067$; SRMR $=.047]$. Modification indices suggested that the residuals of TG1 and TG2 should be allowed to co-vary. Therefore, in an additional model we added this path and it resulted in a significant improvement in fit $\left[\Delta \chi^{2}(1)=9.33\right.$, $p=.002]$. Rhythm Perception (RP) had a weak but significant loading $(r=.37, p<.001)$ whilst the remaining tasks loaded strongly with an average of $r=.64\left(\mathrm{Min}=.50, \mathrm{Max}=.75, S D_{r}\right.$ $=.09)$.

Next, we confirmed the presence of a memory factor by specifying the three memory tasks to define a single latent $\mathrm{Gm}$ factor. Fit statistics are not available for this model because degrees of freedom are equal to zero. However, all three tasks loaded moderately supporting the presence of latent $\mathrm{Gm}$ : VM $(r$ $=.56), \mathrm{NM}(r=.48)$, and SM $(r=.45)$.

Rammsayer and Brandler extracted a single psychometric $g$ (G) factor from the cognitive measures in their study. Instead, we used a hierarchical model in which specific lower order factors were defined (Gc, Gs, and Gf; see Table 5) as well as $g$. First we attempted to define the lower order factors but statistics showed the model's fit was not adequate $\left[\chi^{2}(51)=107.83\right.$, $p<.001$, CFI $=.885$; RMSEA $=.106$; SRMR $=.072]$. Therefore, in consultation with modification indices, we correlated the residuals of the Series and Matrices tests. This resulted in a significant improvement in fit $\left[\Delta \chi^{2}(1)=32.53, p<.001\right]$, which was now considered adequate $\left[\chi^{2}(50)=75.29, p=.01\right.$, CFI $=.949 ; \mathrm{RMSEA}=.071 ; \mathrm{SRMR}=.059]$. The average loading of the tasks across all factors in this improved solution was $r=.69$ $\left(\min =.51, \max =.86, S D_{r}=.11\right)$ and the correlations between the three first order factors were strong. In a subsequent model the first order factors were used to defined a $g$ factor and the fit of this hierarchical model was also adequate $\left[\chi^{2}(50)=75.29, p\right.$ $=.01, \mathrm{CFI}=.949 ; \mathrm{RMSEA}=.071 ; \mathrm{SRMR}=.059]$. The loading of each factor on $g$ was strong: $\mathrm{Gf}(r=.84)$, Gc $(r=.78)$, Gs $(r$ $=.94)$.

Having confirmed the presence of temporal, memory and psychometric factors, we were able to address the extent to which $\mathrm{Gt}$ and $\mathrm{Gm}$ are related and predict variance in psychometric $g$. To accomplish this, we first ran an unrelated predictor model in which $g$ was regressed onto the independent factors $\mathrm{Gt}$ and Gm. In this model, both Gt $(r=.62)$ and $\mathrm{Gm}(r=.55)$ predicted a significant but comparable amount of variance in $g$. Model statistics showed that the fit was not quite adequate $\left[\chi^{2}(202)=262.99, p<.001, \mathrm{CFI}=.921, \mathrm{RMSEA}=.055\right.$, $\mathrm{SRMR}=.086]$. Therefore, we tested a related predictor model in which $\mathrm{Gm}$ was regressed onto Gt; whilst still maintaining regression paths from each of these to $g$. This related predictor model resulted in a significant improvement in fit $\left[\Delta \chi^{2}(1)=\right.$ $7.24, p=.01 ; \chi^{2}(201)=255.74, p=.005, \mathrm{CFI}=.930, \mathrm{RMSEA}$ $=.052, \mathrm{SRMR}=.069]$.

This hierarchical $\mathrm{g}$ with related predictors model is presented as Figure 2. The relationship between Gt and Gm is moderately strong, with the latent variables sharing approximately $20 \%$ of their variance. This path was necessary for satisfactory fit and its addition resulted in a marked decrease in the size of the coefficient between Gt and $g$ (.47 compared to .62), but not between $\mathrm{Gm}$ and $\mathrm{g}$ (.53 versus .55). The standardized direct effect of Gt on $g$ is .47 and the indirect effect is $.23\left(.425^{*} .531\right)$. Thus, $34 \%$ of the effect of Gt on G appears to reflect memory captured in latent $\mathrm{Gm}$.

In light of the smaller yet significant path between Gt and $g$ in this related predictor model, we defined a model which excluded $g$ and instead regressed each of the lower order factors onto related Gt and Gm factors. The purpose of this was to better understand the moderate relationship between Gt and $g$ after accounting for $\mathrm{Gm}$ functions. The fit of this model was $\operatorname{good}\left[\chi^{2}(197)=244.91, p=.01, \mathrm{CFI}=.938, \mathrm{RMSEA}=.049\right.$, $\mathrm{SRMR}=.067]$. The path from $\mathrm{Gm}$ to $\mathrm{Gc}$ was significant $(r$ $=.57, p<.001)$ but not from Gt to Gc $(r=.22, p=.11)$. The path from Gm to Gs was not significant $(r=.19, \mathrm{p}=.24)$ but it was from Gt to Gs $(r=.55, p<.001)$. Gf was predicted signify- 


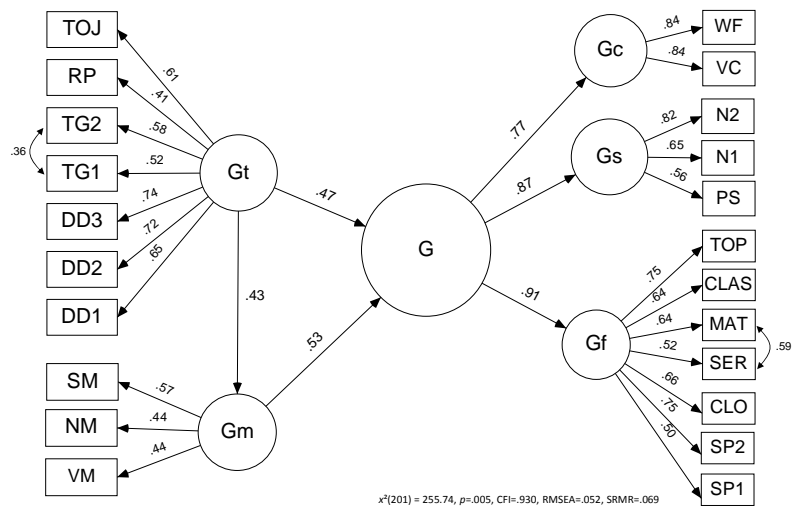

Figure 2.

Hierarchical g model with related Gt and Gm predictors and standardized parameter estimates.

cantly by $\mathrm{Gm}(r=.56, p<.001)$ and to a weaker degree by $\mathrm{Gt}$ $(r=.39, p=.003)$. In a subsequent model we dropped these non-sig- nificant paths, as well as the covariance between Gs and $\mathrm{Gc}$ because of their relative independence. The fit of this model decreased significantly $\left[\Delta \chi^{2}(3)=22.34, p<.001\right]$. However, overall model fit remained statistically adequate $\left[\chi^{2}(200)\right.$ $=267.25, p<.001, \mathrm{CFI}=.913, \mathrm{RMSEA}=.058, \mathrm{SRMR}=.078]$ and in the interest of parsimony, this more restrictive modelshown in Figure 2-should be favoured over the former. As can be seen, $\mathrm{Gt}$ relates strongly to $\mathrm{Gm}\left(R^{2}=.37\right)$ and $\mathrm{Gs}\left(R^{2}=.48\right)$. The relationship between Gt and Gf is markedly weaker and these constructs share only $9 \%$ of variance. Gm on the other hand, relates strongly to both $\mathrm{Gc}\left(R^{2}=.67\right)$ and $\mathrm{Gf}\left(R^{2}=.40\right)$.

\section{Discussion-Study 2}

The reanalysis in Study 2 has provided a more rigorous assessment of the hypothesis that temporal discrimination tasks reflect memory functions than Study 1 because of a larger test battery of cognitive measures. The CFA models show that the relation of latent $\mathrm{Gt}$ to $\mathrm{Gm}$ must be incorporated into these structural models to achieve adequate fit. Moreover, it appears that around $35 \%$ of the relationship between Gt and general intelligence estimates $(g)$ can be explained by memory functions shared with $\mathrm{Gm}$. Of the three broad cognitive factors extracted in the second model (see Figure 3), Gt appeared to relate more strongly to speed of processing (Gs) and Gm than either Gf, or Gc.

\section{General Conclusion}

Recent research has proposed that temporal resolution of the CNS is partly responsible for intelligent functioning and that auditory temporal discrimination tasks provide a valid measure of this resolution (Helmbold \& Rammsayer, 2006; Helmbold, Troche, \& Rammsayer, 2006b; Helmbold et al., 2007b; Rammsayer \& Brandler, 2002, 2004, 2007). This paper has questioned this notion and has presented the results of two studies designed to elicit the nature of the functions underpinning performance on temporal discrimination tasks.

Study 1 showed that the construct measured by the auditory TG task is not modality specific. The correlation between visual and auditory TG was strong; the tasks shared $42 \%$ of variance. Strong relationships have generally not been evident when adapting ECTs across modalities. For example, the relationship between visual IT and auditory IT seldom exceeds $r=.30$ (see

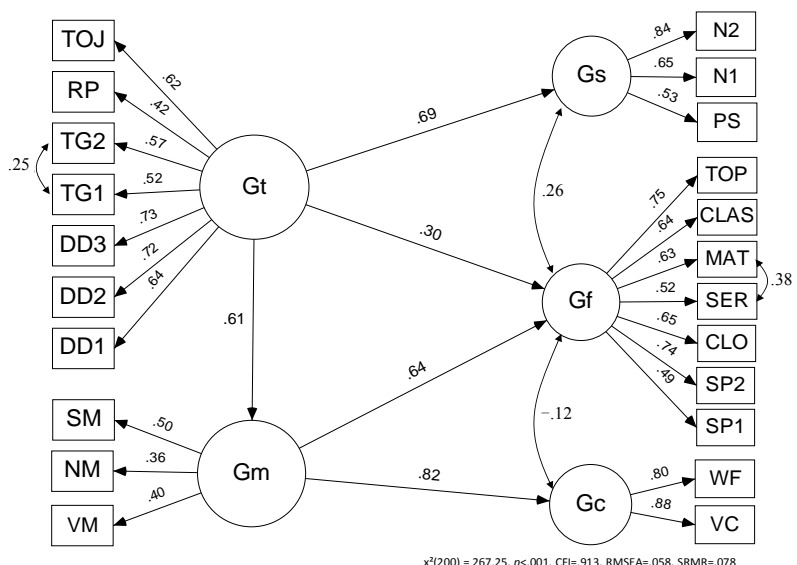

Figure 3.

Broad ability factors model with related Gt and Gm predictors and standardized parameter estimates.

e.g. Deary, 2000) and the variance in these tasks has been largely attributed to peripheral sensory type processes (Burns, Nettelbeck, McPherson, \& Stankov, 2007; Burns, Nettelbeck, \& White, 1998; White, 1996; Zajac \& Burns, 2007). Contrary to this, the strong correlation between visual and auditory TG suggests the processing required by these tasks might not be sensory but rather cognitively based. This would explain the relative independence of sensory and temporal discrimination factors reported previously (Rammsayer \& Brandler, 2004).

Study 1 measured the distinct constructs, Gs and WM, to better understand the observed correlation between TG and intelligence. Speed of processing was measured using traditional speed tasks as well as widely researched ECTs (RT and IT). The impetus for including RT and IT was the proposition that auditory temporal tasks might be analogous to ECTs in terms of providing an estimate of neural efficiency (Helmbold et al., 2007b). The present study does not support this hypothesis. Neither the visual nor auditory TG tasks predicted a statistically significant amount of variance in the composite speed measure with the shared variance near zero.

The regressions of DM-a measure of working memory onto visual and auditory TG tasks were not statistically significant. However, given the near equivalence of the TG tasks across experimental conditions and the consistency of the effect size, the samples were combined across conditions and visual and auditory TG did predict a significant amount of variance in $\mathrm{DM}$; the size of the effect again remained consistent (about $10 \%$ shared variance). This shows that the absence of a significant effect within experimental conditions reflects a lack of statistical power. Future studies should increase sample size to overcome this issue.

The reanalysis of Rammsayer and Brandler's (2007) data in Study 2 provide further evidence that temporal discrimination tasks rely, at least to some extent, on memory functions. In the hierarchical $g$ model (Figure 2), the path between latent Gt and $\mathrm{Gm}$ factors was both necessary and significant, with the latent factors sharing $20 \%$ of their variance. Furthermore, it was found that around 35\% of Gt's relationship to $g$ could be attributed to memory functions represented by latent $\mathrm{Gm}$. In the second model (Figure 3), the regression of Gm on Gt was stronger, with the constructs sharing around $37 \%$ of their variance. Of the three broad cognitive ability factors defined, Gt was most strongly related to Gs. This finding is somewhat consistent with earlier studies in which Gt has been found to share 
variance with RT factors. The analysis in earlier studies, however, has been framed to explore which of Gt and RT explains more $g$ variance. Not surprisingly, Gt emerges as the stronger predictor and it almost wholly accounts for the relationship between RT and $g$ (Helmbold \& Rammsayer, 2006; Helmbold et al., 2007b; Rammsayer \& Brandler, 2007).

In the hierarchical model (Figure 2) in Study 2 it appeared that Gt measured functions over and above memory, which predicted $g$ variance. The non-hierarchical model (Figure 3) shows, however, that this significant Gt $\times g$ path essentially reflects Gt's relationship to Gs, and it is likely because of this relationship that Gt can account for the correlation between RT and $g$. This finding does not imply that Gt measures anything more fundamental to intelligence than RT tasks; it simply suggests that they measure the same functions.

The relationship reported herein between Gt and memory measures are consistent with the requirements of the temporal tasks. Duration Discrimination (DD) requires internal representations of timed intervals to remain accessible for several seconds following their presentation; TG requires accurate learning - and thus memorizing - of a standard stimulus, as well as accurate retrieval of the learnt standard and comparisons with trial stimuli. Interestingly, the pitch and line-length discrimination tasks in Study 1 had the same requirements as TG but did not contribute substantially to the prediction of the working memory task. One explanation for this finding is that functions involved in TG are more complex than for pitch and line-length tasks. Auditory sensory memory, for instance, can retain information concerning dimensions like intensity and frequency for four-to-ten seconds (Jaaskelainen, Hautamake, Naatanen, \& Ilmoniemi, 1999). In the pitch task, then, it is plausible that the comparisons of stimuli rely heavily on these sensory memory traces. Conversely, time is not a perceptual dimension but a cognitively derived entity (Michon, 1990) and therefore the comparisons of stimuli in auditory and visual TG tasks rely on cognitive representations of the durations which appear to be memorised and rehearsed.

Previous research supports this hypothesis. It has been found in a number of studies that temporal processing of durations involves the prefrontal cortex (Elbert, Ulrich, Rockstroth, \& Lutzenberger, 1991; Harrington, Haaland, \& Knight, 1998) which is the brain region thought to play a critical role in the distributed neural systems which achieve working memory (Engle, Kane, \& Tuholski, 1999; Gibbons et al., 2002). Furthermore, an event-related potential (ERP) study which compared temporal and pitch discrimination tasks showed enhanced prefrontal activation in the temporal task (Gibbons et al., 2002). This finding was interpreted as indicating a much stronger contribution of executive memory functions to temporal as opposed to pitch discrimination and it was concluded that "to perceive time and to evaluate temporal properties of a given stimulus, formation of cognitive temporal representations is required-a process primarily based on executive working memory functions" (Gibbons et al., 2002: p. 963).

In summary, the findings herein and those of previous studies raise questions regarding the extent to which auditory temporal discrimination tasks should be considered measures of neural efficiency and by extension, temporal resolution of the CNS. It appears that the observed relationship between auditory temporal discrimination tasks and measures of $g$ may be explained almost entirely in terms of memory functions and speed of processing. More specifically, temporal discrimination performance is confounded by both memory and speed functions and its relationship to the latter does not automatically imply that temporal resolution of the CNS is involved. Even if temporal resolution of the CNS is independent of higher order cognitive operations (Rammsayer \& Brandler, 2002: p. 509) temporal discrimination tasks are not. Attempts to gauge the strength of the relationship between CNS resolution and intelligence-if indeed there is one- using such tasks will not be unequivocal.

\section{References}

Breitmeyer, B. G. (2007). Visual masking: Past accomplishments, present status, future developments. Advances in Cognitive Psychology, 3, 9-20. doi:10.2478/v10053-008-0010-7

Burns, N. R., \& Nettelbeck, T. (2003). Inspection time in the structure of cognitive abilities: Where does IT fit? Intelligence, 31, 237-255. doi:10.1016/S0160-2896(02)00120-4

Burns, N. R., Nettelbeck, T., \& Cooper, C. (2000). Event related potential correlates of some human cognitive ability constructs. Personality and Individual Differences, 29, 157-168. doi:10.1016/S0191-8869(99)00184-1

Burns, N. R., Nettelbeck, T., \& McPherson, J. (2009). Attention and intelligence: A factor analytic study. Journal of Individual Differences, 30, 44-57. doi:10.1027/1614-0001.30.1.44

Burns, N. R., Nettelbeck, T., McPherson, J., \& Stankov, L. (2007). Perceptual learing on inspection time and motion perception. Journal of General Psychology, 134, 83-100. doi:10.3200/GENP.134.1.83-100

Burns, N. R., Nettelbeck, T., \& White, M. (1998). Testing the interpretation of inspection time as a measure of speed of sensory processing. Personality and Individual Differences, 24, 25-39. doi:10.1016/S0191-8869(97)00142-6

Carroll, J. B. (1993). Human cognitive abilities: A survey of factoranalytic studies. Cambridge, New York: Cambridge University Press. doi:10.1017/CBO9780511571312

Cattell, R. B. (1961). Culture free intelligence test, scale 3. Champaign, IL: Institute for Personality and Ability Testing.

Deary, I. J. (2000). Looking down on human intelligence; from psychometrics to the brain. New York: Oxford University Press.

Elbert, T., Ulrich, R., Rockstroth, B., \& Lutzenberger, W. (1991). The processing of temporal intervals reflected by CNV-loke brain intervals. Psychophysiology, 28, 648-655. doi:10.1111/i.1469-8986.1991.tb01009.x

Engle, R. W., Kane, M. J., \& Tuholski, S. W. (1999). Individual differences in working memory capacity and what they tell us about controlled attention, general fluid intelligence, and functions in the prefrontal cortex. In A. Miyake, \& P. Shah (Eds.), Models of working memory: Mechanisms of active maintenance and executive control (pp. 102-134). Cambridge: Cambridge University Press.

Evans, G., \& Nettelbeck, T. (1993). Inspection time: A flash mask to reduce apparent movement effects. Personality and Individual Differences, 15, 91-94. doi:10.1016/0191-8869(93)90045-5

Gibbons, H., Brandler, S., \& Rammsayer, T. H. (2002). Dissociating aspects of temporal and frequency processing: A functional ERP in humans. Cortex, 39, 947-965. doi:10.1016/S0010-9452(08)70872-4

Grudnik, J. L., \& Kranzler, J. H. (2001). Meta-analysis of the relationship between inspection intelligence and inspection time. Intelligence, 29, 523-535. doi:10.1016/S0160-2896(01)00078-2

Harrington, D. L., Haaland, K. Y., \& Knight, R. T. (1998). Cortical networks underlying mechanisms of time perception. Journal of Neuroscience, 18, 1085-1095.

Helmbold, N., \& Rammsayer, T. (2006). Timing performance as a predictor of psychometric intelligence as measured by speed and power tests. Journal of Individual Differences, 27, 20-37. doi:10.1027/1614-0001.27.1.20

Helmbold, N., \& Rammsayer, T. H. (2006). Timing performance as a predictor of psychometric intelligence as measured by speed and power tests. Journal of Individual Differences, 27, 20-37. doi:10.1027/1614-0001.27.1.20

Helmbold, N., Troche, S., \& Rammsayer, T. (2006a). Temporal information processing and pitch discrimination as predictors of general intelligence. Canadian Journal of Experimental Psychology, 60, 294-306. doi:10.1037/cjep2006027 
Helmbold, N., Troche, S., \& Rammsayer, T. (2007a). Processing of temporal and nontemporal information as predictors of psychometric intelligence: A structural-equation-modeling approach. Journal of Personality, 75, 985-1005. doi:10.1111/j.1467-6494.2007.00463.x

Helmbold, N., Troche, S. J., \& Rammsayer, T. H. (2006b). Temporal information processing and pitch discrimination as predictors of general intelligence. Canadian Journal of Experimental Psychology, 60, 294-306. doi:10.1037/cjep2006027

Helmbold, N., Troche, S. J., \& Rammsayer, T. H. (2007b). Processing of temporal and nontemporal information as predictors of psychometric intelligence: A structural-equation-modeling approach. Journal of Personality, 75, 985-1005. doi:10.1111/j.1467-6494.2007.00463.x

Hendrickson, A. E. (1982). The biological basis of intelligence part I: Theory. In H. J. Eysenck (Ed.), A model for intelligence (pp. 151-196). Berlin: Springer-Verlag.

Hendrickson, D. E. (1982). The biological basis of intelligence part II: Measurement. In H. J. Eysenck (Ed.), A model for intelligence (pp. 197-230). Berlin: Springer-Verlag.

Hick, W. E. (1952). On the rate of gain of information. Quarterly Journal of Experimental Psychology, 4, 11-26. doi:10.1080/17470215208416600

Horn, W. (1983). Leistungsprufsystem. Göttingen, Germany: Hogrefe.

Jaaskelainen, I. P., Hautamake, M., Naatanen, R., \& Ilmoniemi, R. J. (1999). Temporal span of human echoic memory and mismatch negativity: Revisited. Neuroreport, 10, 1305-1308. doi:10.1097/00001756-199904260-00028

Jäger, A. O., Süß, H.-M., \& Beauducel, A. (1997). Berliner intelligenzstruktur test form 4. Göttingen, Germany: Hogrefe.

Jensen, A. R. (1982). Reaction time and psycyometric g. In H. J. Eysenck (Ed.), A model for intelligence (pp. 93-132). Berlin: Springer.

Jensen, A. R. (2005). Mental chronometry and the unification of differential psychology. In R. J. Sternberg \& J. E. Pretz (Eds.), Cognition and Intelligence: Identifying the mechanisms of the mind (pp. 26-50). Cambridge: Cambridge University Press.

Jensen, A. R. (2006). Clocking the mind: Mental chronometry and individual differences. Amsterdam: Elsevier.

Kyllonen, P. C., \& Christal, R. E. (1990). Reasoning ability is (little more than) working-memory capacity?! Intelligence, 14, 389-433. doi:10.1016/S0160-2896(05)80012-1

McPherson, J., \& Burns, N. R. (2005). A speeded coding task using a computer-based mouse response. Behavior Research Methods, 37, 538-544. doi:10.3758/BF03192725

Michon, J. A. (1990). Implicit and explicit representations of time. In R. A. Block (Ed.), Cognitive models of psychological time (pp. 37-58). Hillsdale, NJ: Lawrence Erlbaum Associates.

Muthen, L. K., \& Muthen, B. O. (1998). Mplus. The comprehensive modelling program for applied research. User's guide. Los Angeles: Muthen and Muthen.

Nettelbeck, T. (1987). Inspection time and intelligence. In P. A. Vernon (Ed.), Speed of information processing and intelligence (pp. 295-346). Norwood, NJ: Ablex.

Nettelbeck, T. (2001). Correlation between inspection time and psychometric abilities. A personal interpretation. Intelligence, 29, 459-474. doi:10.1016/S0160-2896(01)00072-1

Nettelbeck, T. (2003). Inspection time and g. In H. Nyborg (Ed.), The scientific study of general intelligence: Tribute to Arthur R. Jensen (pp. 77-92). Amsterdam, Boston: Permagon.

Neubauer, A. C., \& Fink, A. (2005). Basic information processing and the psychophysiology of intelligence. In R. J. Sternberg, \& J. E. Pretz (Eds.), Cognition and intelligence: Identifying the mechanisms of the mind (pp. 68-87). Cambridge: Cambridge University Press.

O’Connor, T. A., \& Burns, N. R. (2003). Inspection time and general speed of processing. Personality and individual differences, 35 , 713-724. doi:10.1016/S0191-8869(02)00264-7

Petrill, S. A., Luo, D., Thompson, L. A., \& Detterman, D. K. (2001). Inspection time and the relationship among elementary cognitive tasks, general intelligence, and specific cognitive abilities. Intelligence, 29, 487-496. doi:10.1016/S0160-2896(01)00074-5

Rammsayer, T. H., \& Brandler, S. (2002). On the relationship between general fluid intelligence and psychophysical indicators of temporal resolution in the brain. Journal of Research in Personality, 36, 507-530. doi:10.1016/S0092-6566(02)00006-5

Rammsayer, T. H., \& Brandler, S. (2004). Aspects of temporal information processing: A dimensional analysis. Psychological Research, 69, 115-123. doi:10.1007/s00426-003-0164-3

Rammsayer, T. H., \& Brandler, S. (2007). Performance on temporal information processing as an index of general intelligence. Intelligence, 35, 123-139. doi:10.1016/j.intell.2006.04.007

Reed, T., \& Jensen, A. (1993). Choice reaction time and visual pathway nerve conduction velocity both correlate with intelligence but appear not to correlate with each other: Implications for information processing. Intelligence, 17, 191-203. doi:10.1016/0160-2896(93)90027-3

Sheppard, L. D., \& Vernon, P. A. (2008). Intelligence and speed of information-processing: A review of 50 years of research. Intelligence, 44, 535-551.

Stankov, L. (2005). Reductionism versus charting. In R. J. Sternberg \& J. E. Pretz (Eds.), Cognition and intelligence: Identifying the mechanisms of the mind (pp. 51-67). Cambridge: Cambridge University Press.

Surwillo, W. W. (1968). Timing of behaviour in senescence and the role of the central nervous system. In G. A. Talland (Ed.), Human aging and behavior (pp. 1-35). New York: Academic Press.

Thurstone, L. L. (1938). Primary mental abilities. Chicago, IL: University of Chicago Press.

Vernon, P. A. (1993). Intelligence and neural efficiency. In D. K. Detterman (Ed.), Current topics in human intelligence (Vol. 3, pp. 171-188). New Jersey: Ablex Publishing Corporation.

Weiss, R. H. (1971). Grundintelligenztest CFT 3 skala 3. Braunschweig, Germany: Westermann.

Wetherill, G. B., \& Levitt, H. (1965). Sequential estimation of points on a psychometric function. British Journal of Mathematical and Statistical Psychology, 18, 1-10. doi:10.1111/j.2044-8317.1965.tb00689.x

White, M. (1996). Interpreting inspection time as a measure of the speed of sensory processing. Personality and Individual Differences, 20, 351-363. doi:10.1016/0191-8869(95)00171-9

Zajac, I. T., \& Burns, N. R. (2007). Measuring auditory inspection time in primary school children. Journal of Individual Differences, 28 45-52. doi:10.1027/1614-0001.28.1.45 


\section{Appendix A}

Frequency distribution of stimuli in the stimuli set presented within the Dissociation Paradigm.

\begin{tabular}{|c|c|c|c|c|c|c|c|c|c|}
\hline \multirow[b]{2}{*}{ Dimension 2} & \multirow[t]{2}{*}{ Dimension 1} & \multicolumn{7}{|c|}{ Stimulus Duration } & \multirow{2}{*}{$\Sigma$} \\
\hline & & $125 \mathrm{~ms}$ & $150 \mathrm{~ms}$ & $175 \mathrm{~ms}$ & $200 \mathrm{~ms}(\mathrm{~S})$ & $225 \mathrm{~ms}$ & $250 \mathrm{~ms}$ & $275 \mathrm{~ms}$ & \\
\hline \multirow{8}{*}{$\begin{array}{l}\text { Auditory } \\
\text { (Pitch) }\end{array}$} & $964 \mathrm{~Hz}$ & 1 & 1 & 1 & 3 & 1 & 1 & 1 & 9 \\
\hline & $976 \mathrm{~Hz}$ & 1 & 1 & 1 & 3 & 1 & 1 & 1 & 9 \\
\hline & $988 \mathrm{~Hz}$ & 1 & 1 & 1 & 3 & 1 & 1 & 1 & 9 \\
\hline & $1000 \mathrm{~Hz}(\mathrm{~S})$ & 3 & 3 & 3 & 9 & 3 & 3 & 3 & 27 \\
\hline & $1012 \mathrm{~Hz}$ & 1 & 1 & 1 & 3 & 1 & 1 & 1 & 9 \\
\hline & $1024 \mathrm{~Hz}$ & 1 & 1 & 1 & 3 & 1 & 1 & 1 & 9 \\
\hline & $1036 \mathrm{~Hz}$ & 1 & 1 & 1 & 3 & 1 & 1 & 1 & 9 \\
\hline & $\Sigma$ & 9 & 9 & 9 & 27 & 9 & 9 & 9 & 81 \\
\hline \multirow{8}{*}{$\begin{array}{c}\text { Visual } \\
\text { (Line Length) }\end{array}$} & $7 \mathrm{~cm}$ & 1 & 1 & 1 & 3 & 1 & 1 & 1 & 9 \\
\hline & $8 \mathrm{~cm}$ & 1 & 1 & 1 & 3 & 1 & 1 & 1 & 9 \\
\hline & $9 \mathrm{~cm}$ & 1 & 1 & 1 & 3 & 1 & 1 & 1 & 9 \\
\hline & $10 \mathrm{~cm}(\mathrm{~S})$ & 3 & 3 & 3 & 9 & 3 & 3 & 3 & 27 \\
\hline & $11 \mathrm{~cm}$ & 1 & 1 & 1 & 3 & 1 & 1 & 1 & 9 \\
\hline & $12 \mathrm{~cm}$ & 1 & 1 & 1 & 3 & 1 & 1 & 1 & 9 \\
\hline & $13 \mathrm{~cm}$ & 1 & 1 & 1 & 3 & 1 & 1 & 1 & 9 \\
\hline & $\Sigma$ & 9 & 9 & 9 & 27 & 9 & 9 & 9 & 81 \\
\hline
\end{tabular}

Note: $\mathrm{S}=$ Standard 